\title{
Recovery of Silk Sericin from Soap-Alkaline Degumming Solution
}

\author{
Yesol Yang ${ }^{1}$, Sang Mi Lee ${ }^{1}$, Han Sol Lee ${ }^{1}$, and Ki Hoon Lee ${ }^{1,2,3 *}$ \\ ${ }^{I}$ Department of Biosystems \& Biomaterials Science and Engineering, Seoul National University, Seoul 151-921, Korea \\ ${ }^{2}$ Center for Food and Bioconvergence, Seoul National University, Seoul 151-921, Korea \\ ${ }^{3}$ Research Institute for Agriculture and Life Sciences, Seoul National University, Seoul 151-921, Korea
}

\section{Abstract}

Sericin is usually abandoned after the degumming process. However, it could be a valuable bioresource if an economically efficient recovery process could be set up. In this study, sericin was recovered directly from the degummed waste solution by adding calcium chloride, which induced the precipitation of the surfactant, sodium oleate, by charge interaction. The recovery yield was maximum when $10 \%$ of calcium chloride was added. Further increase in the calcium chloride concentration induced the precipitation of sericin. The recovered sericin had a molecular weight distribution similar to that of the hot-water-extracted sericin; but some highmolecular-weight sericin could not be recovered. The secondary structure and amino acid composition of the recovered sericin were similar to those of conventional hot-water-extracted sericin. We expect that sericin recovered from the degummed waste solution could be an alternative to the hot-water-extracted sericin, which is widely used in various applications.

Received : 28 Aug 2013 Accepted : 10 Sep 2013

Keywords:

Silk,

Sericin,

Waste recovery,

Surfactant

\section{Introduction}

Sericin can be used as a polymeric material and its application in various fields has recently received attention (Aramwit et al., 2012; Kundu et al., 2008). In most cases, sericin is extracted by boiling the silkworm cocoon in water (Oh et al., 2011, Kim et al., 2012; Kwak et al., 2013) or in sodium carbonate solution (Yun et al., 2013). The solutions are collected after removing fibroin, and filtered or dialyzed against water. The separated fibroin is generally not considered for further use because of its uncertain quality, as it is possibly damaged during sericin extraction.
Considering that a cocoon is made of approximately $25 \%$ sericin and fibroin making the remaining $75 \%$ is abandoned, these methods are highly inefficient process .

The majority of sericin is extracted during silk textile production, which is the major application of silk cocoons. However, it is treated as a waste product. There have been various reports on sericin recovery. Fabiani et al. (1996) recovered sericin from the degumming process by a membrane technique. They recovered more than $97 \%$ of sericin; however, their degumming process was far different from the one used in the silk industry. They employed a vapor degumming process

\section{*Corresponding author.}

Ki Hoon Lee

Department of Biosystems \& Biomaterials Science and Engineering, Seoul National University 1 Gwanak-ro, Gwanak-gu, Seoul 151-921, Korea

Tel: +82-2-880-4625 / FAX: +82-2-873-2285

E-mail: prolee@snu.ac.kr 
using only water, without a surfactant. Gulrajani et al. (2009) also applied a membrane technique to recover sericin from the degummed waste solution. However, the purity of sericin was low because they did not separate the surfactant from sericin. Vaithanomsat and Kitpreechavanich (2008) also used membrane filtration for the recovery of sericin; however, the molecular weight of sericin was only 2-9 $\mathrm{kDa}$, which is inadequate for polymeric applications. Capar (2012) and Capar et al. (2008) recovered sericin from the cocoon cooking wastewater. However, the concentration of sericin in the cocoon cooking wastewater was only $2-3 \mathrm{~g} / \mathrm{L}$, whereas approximately $10 \mathrm{~g} / \mathrm{L}$ of sericin is expected from the degummed waste solution if the liquor ratio is 1:25 during degumming. This indicates that the cocoon cooking wastewater is not the best source for sericin recovery. Lin et al. (2012) recovered sericin by adding quaternized chitosan; however, the coagulant so obtained contained both chitosan and sericin. Though the recovery of sericin from the degumming waste solution has been investigated, but the removal of the surfactant was problematic. During degumming, sodium oleate (also known as Marseille soap ) is added as the surfactant and sericin is isolated from the solution in order to prevent recontamination. The interaction between sericin and sodium oleate is hydrophobic interaction. Capar et al. (2009) reported that low-temperature crystallization is highly efficient for the removal of surfactant from the degummed waste solution. By adjusting $\mathrm{pH}$ to 3.5 , the surfactant is split into free fatty acid and salt. The free fatty acids are then crystallized by lowering the temperature to $4{ }^{\circ} \mathrm{C}$, while sericin remains in the supernatant. However, this process would be difficult to apply on a large scale, because it consumes very large amount of energy for lowering the temperature.

In order to recover high-purity sericin from the degumming waste solution, the surfactant must be separated from sericin. Calcium chloride is known to form electrostatic interactions with surfactants such as sodium oleate (Pereira et al., 2012). Once the complex is formed, the surfactant loses its potency; this is why soaps do not work well in "hard" water. This concept inspired our idea to add calcium chloride into the degummed waste solution, as this should weaken the function of the surfactant, thereby separating it from sericin.

We added calcium chloride to the degummed waste solution resulting from silk processing in order to isolate the surfactant from sericin. The effect of calcium chloride was verified by measuring the amount of the precipitate obtained and the purity of sericin. In addition, the molecular weight distribution, secondary structure, and amino acid composition were analyzed to characterize the recovered sericin.

\section{Materials and Methods}

\section{Materials}

Silk cocoons were obtained from the National Academy of Agricultural Sciences, Korea . Sodium oleate was purchased from Junsei, Japan, and calcium chloride was purchased from Samchun, Korea. All other chemicals were purchased from Sigma-Aldrich, Korea.

\section{Methods}

\section{Degumming of cocoon}

Each cocoon was cut into eight equivalent pieces and immersed into a degumming solution consisting of $0.3 \mathrm{wt} \%$ sodium oleate and $0.2 \mathrm{wt} \%$ sodium carbonate. Degumming, with rate $28.1 \%$, was performed at $100^{\circ} \mathrm{C}$ for $1 \mathrm{~h}$. Next, the solution was filtered with a nonwoven filter in order to remove the degummed silk fibroin. The solution was left to stand, allowing it to cool down to room temperature.

\section{Isolation of surfactant from sericin}

Calcium chloride was added into the degummed waste solution to achieve a final concentration of 5, 10, and 15 $\mathrm{wt} \%$. Each solution was stirred for $3 \mathrm{~h}$ at room temperature, and then centrifuged at $10,000 \mathrm{~g}$ for $20 \mathrm{~min}$. The supernatant and precipitate were separated for further analysis. The supernatant was dialyzed against distilled water for $3 \mathrm{~d}$ using a dialysis tube (MWCO 6-8 kDa, Spectrum Laboratories, Inc., USA). The dialyzed sericin solution was freeze-dried to obtain the protein in the solid form. The precipitate was dried at $50^{\circ} \mathrm{C}$ for overnight. 


\section{Instrumental analysis}

The transmittance of the supernatant was measured using a UV/Vis spectrometer (Optizen 2120 UV, Mecasys, USA) at $550 \mathrm{~nm}$. Absorbance at $280 \mathrm{~nm}$ was also determined in order to evaluate the protein content in the dialyzed sericin solution. The final yield of the recovered sericin was calculated by measuring the final solid content of the dialyzed sericin solution using a water content analyzer (MB45, Ohaus, Switzerland). The FT-IR spectra of the precipitate were obtained using an ATRFTIR spectrometer (Nicolet 6700, Thermo Scientific, USA). In order to evaluate the molecular weight distribution, amino acid composition, and secondary structure of the recovered sericin, the supernatants were dialyzed and freeze-dried prior to measurement. The molecular weight distribution of sericin was measured using GPC (ÄKTA purifier, GE-Healthcare, USA). The samples were dissolved in and eluted with $4 \mathrm{M}$ urea solution. The amino acid composition of the sericin samples was analyzed using HPLC (HP1100, Hewlett Packard, USA). Each sample was hydrolyzed using $6 \mathrm{~N}$ hydrochloric acid with 0.02 vol $\%$ 2-mercaptoethanol at $110^{\circ} \mathrm{C}$ for $24 \mathrm{~h}$ in a nitrogen-filled vial. The final hydrolysates were dissolved in $\mathrm{pH} 2.2$ sodium citrate buffers and analyzed using HPLC with an Inno-C18 column. The column temperature was $40^{\circ} \mathrm{C}$ and the flow rate was $1 \mathrm{~mL} / \mathrm{min}$. The secondary structure of sericin was analyzed using ATR-FTIR.

\section{Results}

Calcium chloride was added to the degummed solution in order to precipitate the surfactant, sodium oleate. Upon addition of calcium chloride, precipitates were formed in all samples. The precipitates were removed from the solution by centrifugation. Fig. 1 shows the transmittance of the supernatant when different amounts of calcium chloride were added. The transmittance decreased significantly upon addition of $15 \mathrm{wt} \%$ calcium chloride, indicating sufficient surfactant removal. Fig. 2 shows the weight of the precipitate according to the amount of calcium chloride added. This figure shows that $15 \mathrm{wt} \%$ of calcium chloride induced greatest precipitation and was the most efficient in removing the surfactant. In order to verify the removal of surfactant, we performed FT-IR analyses of the

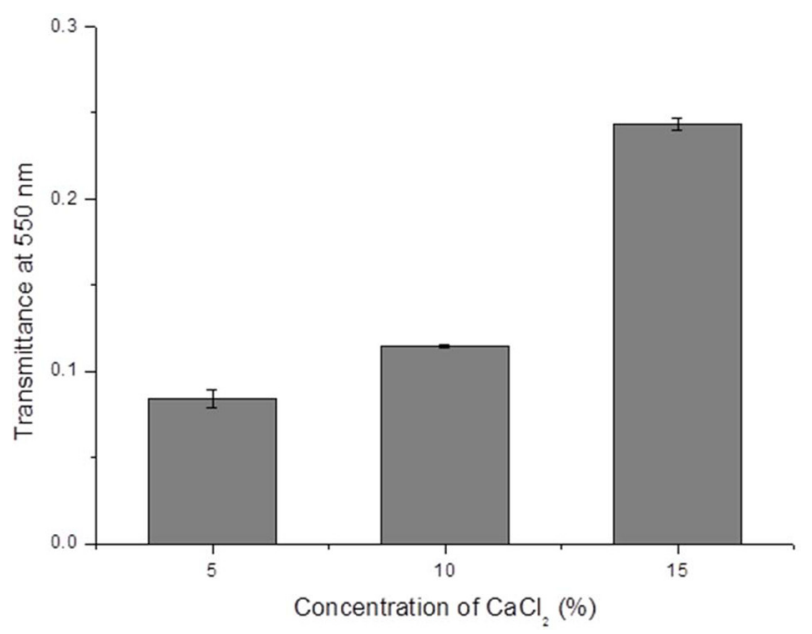

Fig. 1. Transmittance of supernatant resulting from the addition of different amounts of calcium chloride during extraction.

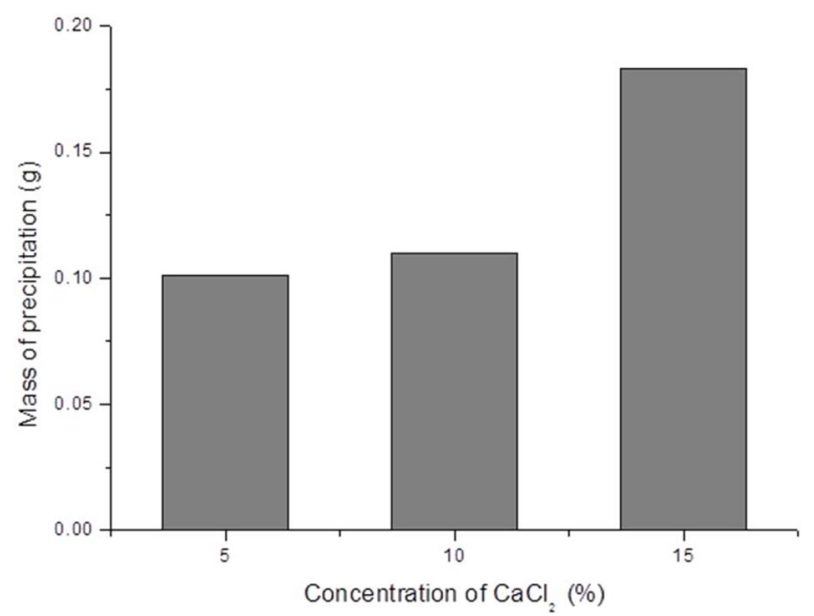

Fig. 2. Weight of precipitate resulting from the addition of different amounts of calcium chloride.

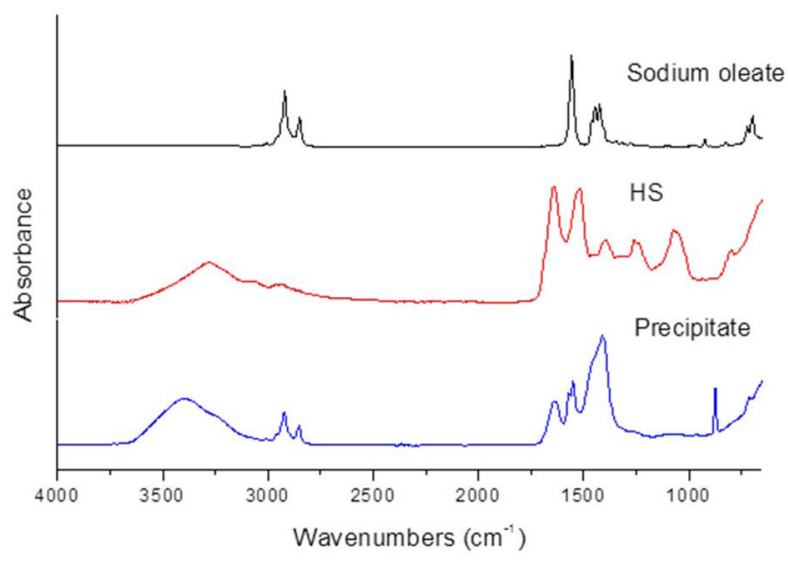

Fig. 3. FT-IR spectra of sodium oleate, hot-water-extracted sericin (HS), and precipitate obtained after adding $10 \mathrm{wt} \%$ of calcium chloride. 


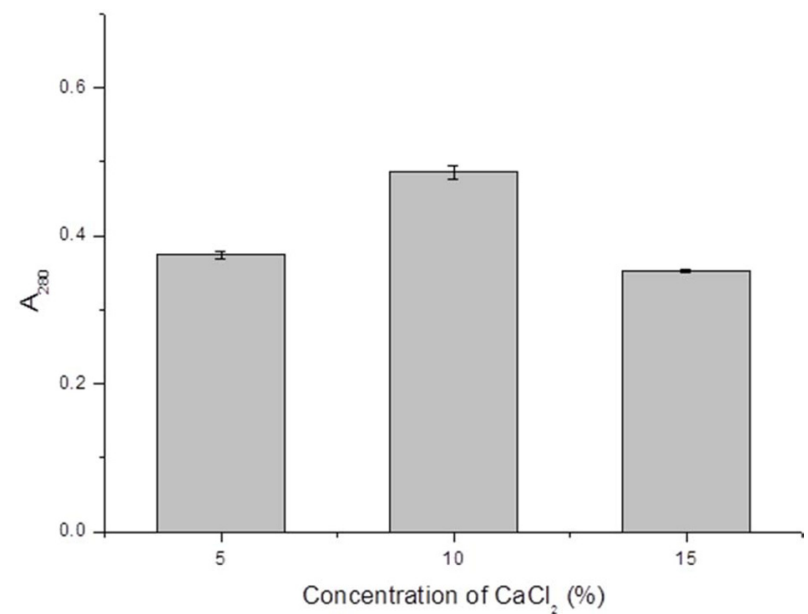

Fig. 4. Absorbance of dialyzed sericin solution at $280 \mathrm{~nm}\left(\mathrm{~A}_{280}\right)$, resulting from the addition of different amounts of calcium chloride.

Table 1. Final yield of sericin recovered from degummed waste solution

\begin{tabular}{|cccc|}
\hline & $\begin{array}{c}\text { Expected } \\
(w t \%)\end{array}$ & $\begin{array}{c}\text { Actual } \\
(w t \%)\end{array}$ & Yield (\%) \\
\hline Concentration & 1.12 & 0.85 & 75.62 \\
\hline
\end{tabular}

precipitates. The FT-IR spectra of the precipitates indicate that they contain not only the surfactant but also sericin (Fig. 3). Proteins show a typical FT-IR spectrum with a strong absorbance peak around $1700-1600 \mathrm{~cm}^{-1}$ due to amide I vibration. This amide I peak can be observed in the spectrum of the precipitate, indicating that sericin is coprecipitated by the addition of calcium chloride. In order to verify the amount of sericin recovered from the degumming solution, the supernatant was dialyzed against distilled water. The amount of sericin in the dialyzed solution was measured by UV spectrometry. The absorbance at $280 \mathrm{~nm}$ $\left(\mathrm{A}_{280}\right)$ indicates that the greatest amount of sericin was recovered when $10 \%$ of calcium chloride was added (Fig. 4 and Table 1). The final yield of sericin is $75.62 \%$. Fig. 5 shows the molecular weight distribution of sericin recovered from the degummed solution. The molecular weight distribution of sericin was similar to that of the hot-water-extracted sericin. The only difference was that the elution time of the maximum intensity peak was delayed, indicating that sericin molecules with a high molecular weight either precipitated during the centrifugation, or degraded during soap-alkaline degumming. The FT-IR spectra of the recovered sericin also showed that there was no significant difference in

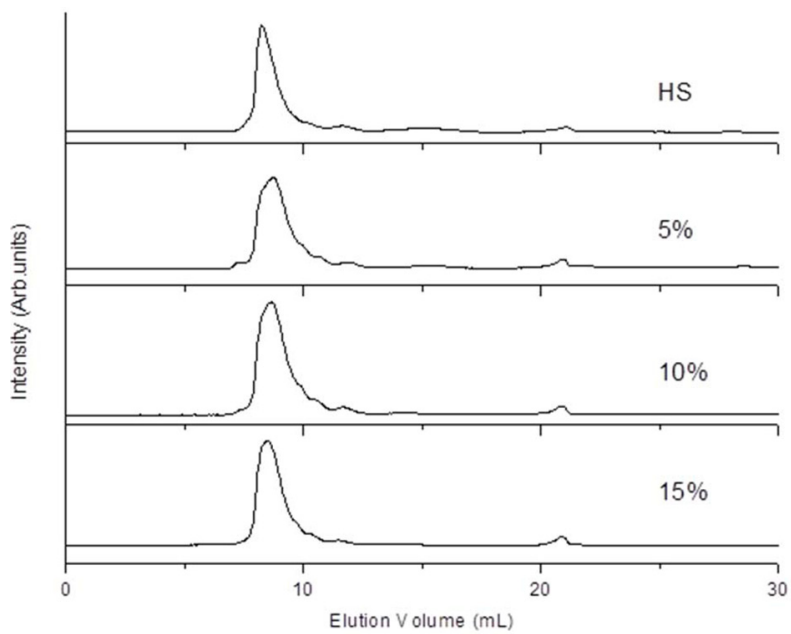

Fig. 5. Molecular weight distribution of sericin recovered from degummed solution with different amounts of calcium chloride added. HS refers to hot-water-extracted sericin.

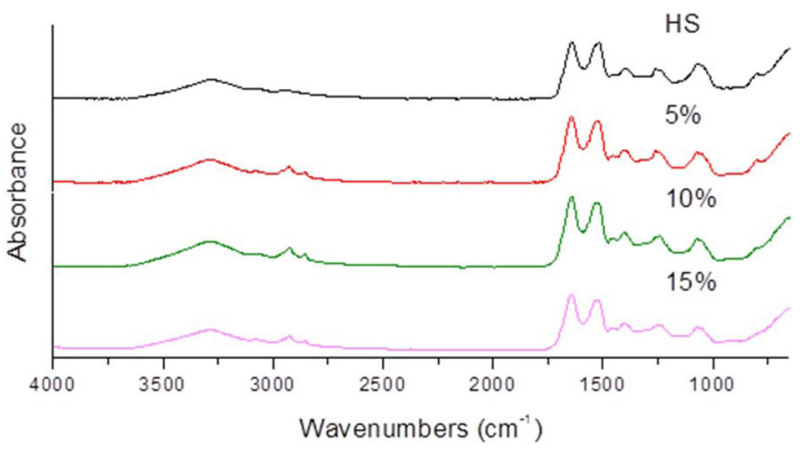

Fig. 6. FT-IR spectra of hot-water-extracted sericin (HS) and recovered sericin, resulting from the addition of different amounts of calcium chloride.

the secondary structures of hot-water-extracted sericin and the sericin recovered using our method (Fig. 6). All sericin samples, whether from hot-water extraction or those recovered via our method, have a random coil structure. In addition, after dialysis, only sericin was detected without the surfactant indicating that surfactant can be removed by the dialysis. Table 2 shows the amino acid composition of the hot-water-extracted and recovered sericin. The data indicate that there is no significant difference in the amino acid composition between the two samples. Therefore, it can be concluded that sodium oleate can be separated from sericin, resulting in the recovery of high-purity sericin from the degummed solution by the addition of calcium chloride. Furthermore, the molecular weight distribution, secondary structure, and amino acid composition of the recovered sericin were similar to those of common hot-water-extracted sericin. 
Table 2. Amino acid composition of hot-water-extracted sericin (HS) and recovered sericin with different amounts of added calcium chloride

\begin{tabular}{|cccccc|}
\hline$(\%)$ & HS $^{c}($ Oh et al. [3]) & $0 \%$ & $5 \%$ & $10 \%$ & $15 \%$ \\
\hline Asx & 13.3 & 14.42 & 14.37 & 14.27 & 14.32 \\
\hline Glx $^{\mathrm{b}}$ & 4.54 & 4.81 & 4.67 & 4.77 & 4.75 \\
\hline Ser & 34.68 & 32.55 & 33.33 & 33.20 & 33.24 \\
\hline His & 0.89 & 0.00 & 1.10 & 1.12 & 1.10 \\
\hline Gly & 18.5 & 12.17 & 12.16 & 11.84 & 12.16 \\
\hline Thr & 7.2 & 7.48 & 7.54 & 7.65 & 7.40 \\
\hline Arg & 5.26 & 4.45 & 4.37 & 4.49 & 4.45 \\
\hline Ala & 4.48 & 5.71 & 5.56 & 5.72 & 5.70 \\
\hline Tyr & 2.97 & 3.69 & 3.70 & 3.78 & 3.76 \\
\hline Val & 2.54 & 6.31 & 6.24 & 6.08 & 6.15 \\
\hline Met & 0.00 & 0.00 & 0.00 & 0.00 & 0.00 \\
\hline Phe & 0.47 & 0.80 & 0.80 & 0.79 & 0.63 \\
\hline Ile & 0.47 & 1.48 & 1.41 & 1.46 & 1.43 \\
\hline Leu & 1.09 & 2.03 & 1.92 & 2.02 & 1.98 \\
\hline Lys & 3.6 & 0.60 & 0.59 & 0.57 & 0.60 \\
\hline Pro & 0.00 & 2.38 & 2.24 & 2.24 & 2.19 \\
\hline
\end{tabular}

${ }^{a}$ Asp + Asn

${ }^{\mathrm{b}} \mathrm{Glu}+\mathrm{Gln}$

${ }^{\mathrm{c}}$ Oh et al., 2011

\section{Discussion}

We added calcium chloride into the degummed solution in order to precipitate the surfactant, sodium oleate. Sodium oleate has one carboxyl group at the end of an alkyl chain, thus allowing the divalent calcium cation to form an electrostatic interaction with it (Pereira et al., 2012). This calcium-oleate complex aggregates into large particles and can be isolated by centrifugation. In order to separate out the aggregates, we also attempted membrane separation; however, severe fouling of the membrane was observed. Our results indicate that the calcium ions precipitate not only sodium oleate but also sericin; this was confirmed by FT-IR analysis of the precipitates. The molar amount of calcium chloride needed to precipitate the entire sodium oleate should be half of the molar amount of the sodium oleate present, because one mole of calcium cation can interact with two moles of oleate. However, in our investigation, we added more than 12 times the required amount of calcium chloride. Because the interaction between sericin and sodium oleate is hydrophobic, we added an excess of calcium chloride to weaken it. However, the addition of calcium chloride precipitated both sericin and the surfactant. In general, high concentrations of ions can induce the salting out of a protein. It seems that the solubility of sericin is reduced at high concentrations of calcium chloride. As a result, less sericin was recovered upon addition of $15 \mathrm{wt} \%$ of calcium chloride than that obtained with addition of $10 \mathrm{wt} \%$, despite the highest transmittance exhibited by the $15 \mathrm{wt} \%$ of calcium chloride after centrifugation .

The recovered sericin should have characteristics similar to those of the hot-water-extracted sericin, which is widely used in material applications. In our study, we compared the molecular weight distribution, secondary structure, and amino acid composition of the recovered and hot-water-extracted sericin. The molecular weight distribution of the recovered sericin was similar to that of the hot-water-extracted sericin except for the early elution times of which indicates that sericin obtained did not have high molecular weight. We have previously shown that high-molecularweight sericin is more hydrophobic than lower weight sericin (Oh et al., 2011). This can contribute to an increased interaction between sodium oleate and sericin, or to the low solubility of highmolecular-weight sericin. In both cases, the high molecular weight sericin would precipitate during centrifugation.

We found no differences in the secondary structure and amino acid compositions of the hot-water-extracted and recovered sericin. The present study shows that the addition of calcium chloride can efficiently separate the surfactant and sericin. Because almost the entire industrial silk degumming process is performed by the alkaline-soap method, our results showing the possibility of recovering sericin from this degummed solution can be advantageous from both economic and environmental perspectives. From the economic point of view, our method of sericin recovery can be applied to the existing procedure. Therefore, there is no need to establish a new process, and the remaining fibroin could also be used for manufacturing textiles and biomaterials. The environmental advantage is that our method would reduce the amount of nitrogenous waste, which is usually disposed of in aqueous ecosystems such as rivers. In our future work, we aim to evaluate the mechanical and biological properties of the recovered sericin. 


\section{Acknowledgement}

This work was supported by the Basic Science Research Program through the National Research Foundation of Korea (NRF), funded by the Ministry of Education (NRF-20100025378).

\section{References}

Aramwit P, Siritientong T, Srichana T (2012) Potential applications of silk sericin, a natural protein from textile industry by-broducts. Waste Manage Res 30, 217-224.

Capar G, Aygun SS, Gecit MR (2008) Treatment of silk production wastewaters by membrane processes for sericin recovery. J Membrane Sci 325, 920-931.

Capar G, Aygun SS, Gecit MR (2009) Separation of sericin from fatty acids towards its recovery from silk degumming wastewaters. J Membrane Sci 342, 179-189.

Capar G (2012) Separation of silkworm proteins in cocoon cooking wastewaters via nanofiltration: Effect of solution $\mathrm{pH}$ on enrichment of sericin. J Membrane Sci 389, 509-521.

Fabiani C, Pizzichini M, Spadoni M, Zeddita G (1996) Treatment of waste water from silk degumming processes for protein recovery and water reuse. Desalination 105, 1-9.

Gulrajani ML, Purwar R, Prasad RK, Joshi M (2009) Studies on structural and functional properties of sericin recovered from silk degumming liquor by membrane technology. J Appl Polym Sci 113, 2796-2804.

Kim MK, Kwak HW, Lee JY, Yun H, Kim MH, Lee KH (2012) Effect of lyoprotectant on the solubility and structure of silk sericin. Int $\mathrm{J}$ Indust Entomol 25, 133-137.

Kundu SC, Dash BC, Dash R, Kaplan DL (2008) Natural protective glue protein, sericin bioengineered by silkworms: Potential for biomedical and biotechnological applications. Prog Polym Sci 33, 998-1102.

Kwak HW, Yang YS, Kim MK, Lee JY, Yun H, Kim MH, Lee KH (2013) Chromium(VI) Adsorption Behavior of Silk Sericin Beads. Int J Indust Entomol 26, 47-53.

Lin J, Wang L, Wang L (2012) Coagulation of sericin protein in silk degumming wastewater using quaternized chitosan. J Polym Environ 20, 858-864.

Oh H., Lee JY, Kim MK, Um IC, Lee KH (2011) Refining hot-water extracted silk sericin by ethanol-induced precipitation. Int J Biol Macromol 48, 32-37.

Pereira RF, Valente AJ, Fernandes M, Burrows HD (2012) What drives the precipitation of long-chain calcium carboxylates (soaps) in aqueous solution. Phys Chem Chem Phys 13, 7517-7527.

Vaithanomsat P, Kitpreechavanich V (2008) Sericin separation from silk degumming wastewater. Sep Purif Technol 59, 129-133.

Yun H, Oh H, Kim MK, Kwak HW, Lee JY, Um IC, Vootla SK, Lee KH (2013) Extraction Conditions of Antherea mylitta Sericin with High Yields and Minimum Molecular Weight Degradation. Int $\mathrm{J}$ of Biol Macromol 52, 59-65. 